\title{
O Presenteísmono âmbito da enfermagem em Unidade de Terapia Intensiva
}

\author{
The presentism in the scope of the nursing in Unit of Intensive Therapy \\ El Presente en el ámbito de la enfermería en Unidad de Terapia Intensiva \\ Luã Gianine Moreira ${ }^{1 *}$, Margareth Fernandes ${ }^{2}$
}

Como citar esse artigo. Moreira, LG; Fernandes, M. O Presenteísmono âmbito da enfermagem em Unidade de Terapia Intensiva. Revista Pró-UniverSUS. 2019 Jan./Jun.; 10 (1): 154-161.

\begin{abstract}
Resumo
O conceito de presenteísmo surge ao reconhecer a importância dos recursos humanos para organização. Novo na literatura da saúde, vem sendo tratado como preocupação dentro do ambiente de trabalho, uma vez que o profissional irá comparecer doente ao trabalho, colocando em risco sua integridade física e principalmente a assistência prestada ao paciente. $\mathrm{O}$ trabalho discute as consequências gerenciais e administrativas do presenteísmo na equipe da UTI. A preocupação sobre o adoecimento dos profissionais que atuam no ambiente de alta complexidade, evita que eles adoeçam e possam causar problemas assistenciais e administrativos para a instituição, melhorando assim a qualidade de vida dos colaboradores. Profissionais da enfermagem que trabalham em Unidades de Terapia Intensiva esbarram a todo tempo nesta problemática, uma vez que precisam trabalhar, mas nem sempre estão em condições psicológicas e físicas para encararem uma jornada de trabalho. Foi realizado uma pesquisa qualitativa descritiva utilizando descritores relacionados ao tema a fim de excluir os demais artigos que não tivessem relação a temática. O foco no presenteísmo torna-se, então, uma estratégia que os enfermeiros gestores e assistencialistas devem adotar para promover o bem-estar, a saúde, melhorar a produtividade, reduzindo assim os custos, ajudando assim na melhoria da assistência prestada.
\end{abstract}

Palavras-chave: Enfermagem; Presenteísmo; UTI.

\begin{abstract}
The concept of presenteeism arises when we recognize the importance of human resources for organization. New in the health literature, it has been treated as a concern within the work environment, since the professional will appear ill at work, putting at risk his or her physical integrity and mainly the care given to the patient. The paper discusses the managerial and administrative consequences of presenteeism in the intensive care unit team. The concern about the illness of the professionals who work in the environment of high complexity, prevents them from becoming ill and can cause administrative and care problems for the institution, thus improving the quality of life of employees. Nursing professionals who work in Intensive Care Units come up against this problem at all times, since they need to work, but they are not always in a psychological and physical condition to face a work day. A qualitative descriptive research was carried out using descriptors related to the theme in order to exclude the other articles that were not related to the theme. The focus on presenteism becomes, then, a strategy that nurses managers and assistentialists must adopt to promote the well- being, health, improving productivity, thus reducing costs, thus helping to improve care delivery.
\end{abstract}

Keywords: Nursing; Presentism; ICU.

\section{Resumen}

El concepto de presentismo surge reconociendo la importancia de los recursos humanos para la organización. Nuevo en la literatura de la salud, se ha tratado como una preocupación dentro del ambiente del trabajo, puesto que el profesional parecerá enfermo al trabajo, poniendo en peligro su integridad física y especialmente la ayuda proporcionada al paciente. El trabajo discute las consecuencias administrativas y administrativos del presentismo en el equipo de ICU. La preocupación por la enfermedad de los profesionales que trabajan en el entorno de alta complejidad, les impide enfermar y pueden causar asistencia y problemas administrativos para la institución, mejorando así la calidad de vida de los empleados. Los profesionales de enfermería que trabajan en unidades de cuidados intensivos se topan con este problema todo el tiempo, ya que necesitan trabajar, pero no siempre están en condiciones psicológicas y físicas para afrontar un día hábil. La investigación cualitativa descriptiva se llevó a cabo utilizando descriptores relacionados con el tema para excluir otros artículos que no estaban relacionados con el tema. El enfoque en presentismo se convierte, entonces, en una estrategia que los gerentes de enfermería y asistencialistas deben adoptar para promover el bienestar, la salud, mejorar la productividad, reduciendo así los costos, ayudando así a mejorar la asistencia proporcionada.

Palabras clave: Enfermería; Presenteísmo; UCI.

1 Enfermeiro. Especialista em Enfermagem em UTI Universidade de Vassouras. RJ, Email: gianine moreira@hotmail.com ORCID: https://orcid.org/0000-0003-3191-1934

2Administradora. Mestre em Ciências Ambientais pela Universidade de Vassouras. Professora do Curso de Pós-graduação de Enfermagem em Terapia Intensiva/UTI Adulto, Univer-

sidade de Vassouras, RJ, Brasil. Email: fernandes.meg@gmail.com ORCID: https://orcid.org/0000-0002-4690-0173 


\section{Introdução}

O conceito de presenteísmo surge ao reconhecer a importância dos recursos humanos para a Organização. Identificado há décadas, mas relativamente novo na literatura da saúde, vem sendo tratado como preocupação dentro do ambiente de trabalho, uma vez que o profissional irá comparecer doente ao trabalho, colocando em risco sua integridade física e principalmente a assistência prestada ao paciente.

O presenteísmo surge ao reconhecer a importância dos recursos humanos para organização. Deriva do termo inglês presenteeism, usado para descrever a relação entre doença e perda de produtividade, consequência de trabalho excessivo e do sentimento de insegurança, resultado do reajuste e estruturação da década de 1990, com altas taxas de desemprego, reestruturação nos setores públicos e privados, diminuição tamanho da organização, redução do número de funcionários público, o aumento do número de pessoas com contratos de trabalho temporários e redução de segurança trabalho.

[...] o presenteísmo passou a ser objeto de estudo na França em meados da década de 1950, a princípio considerado como o absenteísmo de corpo presente, certamente base em comum dos termos relativos aos fatores psicossociais[...]. O interesse científico com o tema emergiu recentemente, no momento em que as empresas passaram a perceber com maior ênfase que tornar-se mais competitivo depende dos colaboradores. ${ }^{1}$

O conceito de presenteísmo foi identificado há décadas, mas é relativamente novo na literatura da saúde

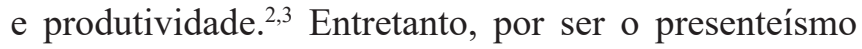
um tema de interesse recente, ainda pode apresentar alguma dificuldade de entendimento e definição. Esta dificuldade, segundo os autores, pode estar relacionada com afirmações de que trabalhar doente representa uma queda de produtividade maior do que o absenteísmo e com ideia de que uma gestão que oferece vantagens aos trabalhadores assíduos gera a competitividade e estimula o presenteísmo. ${ }^{4}$

Ao longo do tempo e até mesmo nos dias de hoje, muitas gerações têm assumido que os trabalhadores são produtivos enquanto estão no trabalho. Porém, os trabalhadores podem estar no trabalho, mas não totalmente focados em suas tarefas devido a problemas de saúde física ou mental. ${ }^{2}$

[...] no Brasil a maioria das pessoas desconhece o conceito que são muitas vezes decorrentes do estresse. O Presenteísmo é pouco diagnosticado e apenas uma pequena parcela busca auxílio médico. As pessoas que comparecem ao trabalho, mesmo sem condições físicas, acabam gerando um círculo vicioso: sem condições físicas ou psíquicas não conseguem cumprir suas tarefas. Sair de casa para ir trabalhar doente, em vez de cumprir repouso ou buscar tratamento médico para maiores cuidados, o medo ou a insegurança de que os gestores pensem que estão com pouca disposição ou sem entusiasmo, isso faz com que a pessoa presenteísta não procure auxílio de tratamento e fique horas depois do horário de expediente para recuperar o tempo perdido, isso faz com que a doença se prolongue, piore ou se transforme em problema crônico. ${ }^{5: 4}$

Para alguns autores existem dois conceitos de Presenteísmo: um envolvendo a saúde ocupacional, relação organizacional e as condições de trabalho, onde o funcionário permanece trabalhando mesmo doente. E o outro, quando os colaboradores permanecem trabalhando além do seu limite, por medo de ser demitido ou excluído por não estar disponível quando necessário. ${ }^{4}$

Presenteísmo se traduz em trabalhar enquanto doente. ${ }^{6,4,7} \mathrm{O}$ presenteísmo surge, como um grave problema, tendo em vista as consequências que dele podem vir: os custos visíveis e invisíveis, o reflexo sobre a produtividade e principalmente sobre a saúde do trabalhador. Contudo, no Brasil, poucas pesquisas tiveram o presenteísmo como objeto de estudo, sugerindo, assim, lacunas que podem ser preenchidas com o aprofundamento deestudos. ${ }^{1}$

O trabalho tem por objetivo discutir as consequências gerenciais e administrativas do presenteísmo na equipe da UTI. Tem-se como justificativa a preocupação sobre $\mathrm{o}$ adoecimento dos profissionais que atuam no ambiente de alta complexidade, evitando assim que eles adoeçam e possam causar problemas assistenciais e administrativos para a instituição, melhorando assim a qualidade de vida dos colaboradores.

O método utilizado foi uma pesquisa qualitativa descritiva utilizando descritores relacionados ao tema a fim de excluir os demais artigos que não tivessem relação a temática.

\section{Materiais e métodos}

Os materiais e métodos utilizados foram os referenciais teóricos através de uma pesquisa qualitativa descritiva utilizando descritores relacionados ao tema: enfermagem; presenteísmo e UTI a fim de excluir os demais artigos que não tivessem relação a temática.

A pesquisa qualitativa não se preocupa com representatividade numérica, mas, sim, com o aprofundamento da compreensão de um grupo social, de uma organização, ${ }^{8,9}$ afirmam:

[...] Os pesquisadores que utilizam os métodos qualitativos buscam explicar o porquê das coisas, exprimindo o que convém ser feito, mas não quantificam os valores e as trocas simbólicas nem se submetem à prova de fatos, pois os dados analisados são não-métricos (suscitados e de interação) e se valem de diferentes abordagens. Na pesquisa qualitativa, o cientista é ao mesmo tempo o sujeito e o objeto de suas pesquisas. O desenvolvimento da pesquisa é imprevisível. 
O conhecimento do pesquisador é parcial e limitado. O objetivo da amostra é de produzir informações aprofundadas e ilustrativas: seja ela pequena ou grande, o que importa é que ela seja capaz de produzir novas informações.

A pesquisa descritiva é definida como pesquisa que realiza o estudo, a análise, o registro e a interpretação dos fatos do mundo físico sem a interferência do pesquisador. ${ }^{10}$

O processo descritivo pode ser descrito coo um estudo de caso onde, após a coleta de dados, realiza-se uma análise das relações entre as variáveis para uma determinar os efeitos resultantes em uma empresa, sistema de produção ou produto. ${ }^{11}$

A revisão integrativa da literatura teve um papel importante pois através desta foi possível fazer uma contextualização da temática com a realidade através de levantamento de dados.

\section{Resultados}

\section{Política Nacional de Saúde do Trabalhador - PNST}

A Política Nacional de Saúde do Trabalhador no SUS é o instrumento definidor da atuação do Sistema Único de Saúde no campo da Saúde do Trabalhador. Assume referenciais da vigilância das condições, dos processos de trabalho, da vigilância epidemiológica dos agravos à saúde deles decorrentes, da promoção, dos produtos e da articulação das ações de cuidado individual às ações coletivas. ${ }^{12}$

Esta política deve possui interfaces com diversas áreas e políticas públicas como Previdência Social, Trabalho e Emprego, Educação, Meio Ambiente, Agricultura, Indústria, Comércio, Mineração, Ciência e Tecnologia, Segurança Pública. ${ }^{13}$

[...] Entre os objetivos da Política está fortalecer a Vigilância em Saúde do Trabalhador, com a identificação das atividades produtivas da população e das situações de risco à saúde; análise das necessidades, demandas e problemas de saúde dos trabalhadores; intervenção nos processos e ambientes de trabalho; produção de tecnologias de intervenção e monitoramento, e controle e avaliação da qualidade dos serviços e programas de saúde do trabalhador. ${ }^{13: s p}$

A política tem como objetivo principal reduzir acidentes e doenças relacionadas ao trabalho, por meio de ações de promoção, reabilitação e vigilância na área de saúde. Suas diretrizes vão compreender a atenção integral à saúde, a articulação intra e intersetorial, a participação popular, o apoio a estudos e a capacitação de recursos humanos. ${ }^{13}$

A operacionalização das diretrizes e estratégias da Política deve ser garantida nos planos de saúde nacional, estadual e municipal, conforme as principais atribuições e responsabilidades de cada nível de gestão do SUS, para o que concorrem os investimentos na qualificação e educação permanente das equipes técnicas e gerenciais e a clara definição dos mecanismos de financiamento. ${ }^{12}$

Sendo assim, a Política Nacional de Saúde do Trabalhador tem por objetivo definir os princípios, as diretrizes e as estratégias a serem observados nas três esferas de gestão do SUS para o desenvolvimento da atenção integral à Saúde do Trabalhador, visando à promoção e a proteção da saúde dos trabalhadores e a redução da morbimortalidade decorrente dos modelos de desenvolvimento e dos processos produtivos. ${ }^{12}$

A saúde é silenciosa, que geralmente não a percebemos em sua plenitude e na maior parte das vezes só percebemos quando adoecemos. A mesma autora também diz que ouvir o próprio corpo é uma boa estratégia para assegurar a saúde com qualidade, pois não existe um limite preciso entre a saúde e a doença, mas uma relação de reciprocidade entre ambas.14 Para ter saúde, é necessário partir da dimensão do ser, pois é nele que ocorrem as definições do normal ou patológico. $\mathrm{O}$ considerado normal em um indivíduo pode não ser em outro; não há rigidez no processo. ${ }^{15}$

[...] Saúde - estado de completo bem-estar físico, mental e social, e não simplesmente à ausência de doença ou enfermidade - é um direito fundamental, e que a consecução do mais alto nível de saúde é a mais importante meta social mundial, cuja realização requer a ação de muitos outros setores sociais e econômicos, além do setor saúde. ${ }^{15: 81}$

Já no Brasil foi discutido, na VII Conferência Nacional de Saúde em 1986, os temas: saúde como direito; reformulação do Sistema Nacional de Saúde (SUS) e financiamento setorial, sendo adotado o seguinte conceito sobre saúde:

[...] em seu sentido mais abrangente, a saúde é resultante das condições de alimentação, habitação, educação, renda, meio ambiente, trabalho, emprego, lazer, liberdade, acesso e posse da terra e acesso a serviços de saúde. É assim, antes de tudo, o resultado das formas de organização social da produção, as quaispodemgerargrandesdesigualdadesnosníveisdevida. ${ }^{15: 81}$

\section{A Síndrome de Burnout}

A Síndrome de Burnout palavra oriunda do inglês, significa exaustão, esvaziamento ou ainda mesmo esgotamento, sendo considerada uma patologia observada entre os profissionais que trabalham na prestação de cuidados, ou seja, acometem na sua grande maioria médicos, enfermeiros e profissionais da educação. ${ }^{16}$

A Síndrome de Burnout não é apresentada na literatura como conceito único, está relacionada a três dimensões próximas, mas independentes: exaustão; despersonalização e diminuição da realizaçãpo profissional. 
Alguns autores argumentam que, "Burnout é um estresse não reconhecido e não resolvido que afeta a dedicação dos indivíduos altamente motivados e que trabalham duro". ${ }^{21: 53}$ Entretanto não se pode afirmar que Burnout seja simplesmente estresse, por que existe uma diferenciação entre os dois conceitos como:

[...] Burnout é a resposta prolongada de estresse,ocorre pela cronificação deste, quando os métodos de enfrentamento falharam ou foram insuficientes. Enquanto estresse pode apresentar pontos positivos e negativos, o Burnout tem características negativas (distresse). Por outro lado, o Burnout está relacionado com o mundo do trabalho, com o tipo de atividades laborais do indivíduo. Além disso, comporta uma dimensão, social, interrelacional, atravésa da despersonalização, o que não necessariamente ocorre com o estresse ocupacional.:22:53

A literatura relaciona as pressões, conflitos de interesse, sobrecarga ao desenvolvimento da síndrome, assim como algumas profissões que são mais susceptíveis ao adoecimento do trabalhador. "[...] as pressões no trabalho, como conflito de interesses e a sobrecarga, contribuem para o desequilíbrio, e o estresse não resolvido leva à deterioração da saúde mental, manifestada por depressão e pela síndrome de Burnout". 23:4

[...] algumas profissões e situações de trabalho têm merecido atenção especial como fonte das tensões que originam a síndrome. Professores, médicos, assistentes sociais e enfermeiros vêm sendo as grandes vítimas dessa síndrome que de forma silenciosa vem provocando muitos estragos na vida desses trabalhadores. ${ }^{22}$

Burnout pode ser também definido como um dos diversos transtornos psíquicos enfrentados pelos enfermeiros, podendo acarretar absenteísmo e consequentemente afastamento do trabalho. Segundo a mesma autora, os índices de transtornos psíquicos na população geral relacionados ao trabalho são preocupantes, devido ao aumento de sua prevalência e aos altos custos sociais. Portanto, os transtornos psíquicos passam a apresentar-secomo um problema de saúde pública, já que podem contribuir com o aumento do número de profissionais afastados do seu ambiente de trabalho pelo INSS. ${ }^{22}$

\section{Estresse / Burnout e suas causas}

A literatura aponta fatores que podem comprometer tanto a integridade mental como a física desses trabalhadores: pressões sofridas durante a jornada de trabalho, a sobrecarga emocional, a carga horária extensa, o cuidado a pacientes críticos, o dimensionamento pessoal, o baixo salário, a falta de reconhecimento profissional. Alguns fatores podem ser vivenciados na rotina diária dos enfermeiros: condições inadequadas de trabalho, despreparo dos profissionais, falta de pessoal, problemas de relacionamento com a equipe, contato direto com pacientes crônicos e familiares. ${ }^{22} \mathrm{O}$ estresse é definido[...] as reações nocivas, física e/ou emocionais, que ocorrem quando as exigências não se igualam à capacidade, aos recursos, ou as necessidades do trabalhador, como resultado da sua interação com as condições de trabalho, o que pode levá-lo a doenças. ${ }^{22: 17}$

Sintomas de fadiga da compaixão: depressão, transtornos de ansiedade, dificuldades de sono, problemas de abuso de substâncias e problemas com relacionamentos, que podem ter um impacto duradouro na saúde física e emocional do enfermeiro. Esse impacto, por sua vez, afeta as organizações e empregadores por meio de erros no trabalho, diminuição da eficiência, e alta rotatividade. ${ }^{22}$

Para as organizações, são significativos o aumento de custos diretos decorrentes do absenteísmo, do incremento da taxa de rotatividade, da queda na eficiência dos trabalhadores, do aumento do número de acidentes de trabalho, por exemplo, e indiretos relacionados à queda na motivação, à desestruturação de relações interpessoais, à insatisfação e à diminuição da qualidade de vida no trabalho. ${ }^{22}$

No mundo globalizado, a enfermagem vem atuando na América Latina com deficiência de recursos humanos e materiais em relação à demanda assistencial. Enumerando alguns fatores percebíveis: deficiência de equipamentos, altas cargas físicas, inadequação na organização dos serviços e nas políticas de pessoal, problemas de relação com a equipe de saúde e com a direção de serviços e deterioração da saúde dos enfermeiros. . $^{22,23,24}$

A fim de assegurar que todos os riscos inerentes aos cuidados em saúde sejam banidos, torna-se necessário para uma assistência de enfermagem mais segura, tanto para o paciente como para o trabalhador, uma assistência de qualidade e correspondendo às expectativas do paciente e família. ${ }^{25}$

\section{Absenteísmo: Entendendo a origem}

A palavra absenteísmo originou-se da palavra "absentismo" aplicado aos proprietários rurais que abandonavam o campo para viver na cidade. Sendo este termo foi aplicado aos trabalhadores que faltavam ao serviço no período industrial. ${ }^{26}$ Os autores citam estudos feitos por Lee e Eriksen de 1990 que indicam o absenteísmo inversamente proporcional à satisfação no trabalho, e a ausência pode ser considerada uma forma de se afastar de pequenas situações indesejáveis. Citam também a observação de fatores como condições de trabalho, natureza da supervisão, estilos de liderança, participação na tomada de decisões e relacionamentos 
profissionais como fatores que podem ajudar a entender o impacto do absenteísmo nas organizações.

Alguns autores destacam a multicausalidade do absenteísmo, pois estudos destacaram a influência da satisfação profissional sobre o absenteísmo. ${ }^{27}$

$\mathrm{Na}$ enfermagem, o absenteísmo merece especial atenção, sobretudo pelas características do trabalho e funcionamento ininterrupto e pelas implicações na redução da equipe e na qualidade da assistência prestada aos pacientes. Afirma também que sua etiologia pode estar relacionada às condições de trabalho, como estilo de liderança e controle, repetitividade das tarefas e falta de integração entre funcionários, que vão interferir indiretamente na assiduidade do trabalhador. As mesmas autoras também denotam que o impacto do trabalho sobre os profissionais da saúde leva a uma sobrecarga mental e física que, aliada à precariedade das condições de trabalho e aos baixos salários, pode desencadear ansiedade, insatisfação, estresse, tensão e, consequentemente, ausências não justificadas ou justificadas por licenças médicas, denominadas de absenteísmo. ${ }^{28}$

Em decorrência do absenteísmo, inevitavelmente têm-se consequências como: queda na qualidade da assistência, sobrecarga de atividades, falta de motivação do profissional ativo, risco na saúde do trabalhador comprometido e aumento dos custos financeiros despendidos com horas extras. Com isto, há necessidade de buscar medidas para prevenir e amenizar as repercussões do absenteísmo bem como os fatores envolvidos na determinação das ausências ao trabalho. Sendo assim, o absenteísmo é considerado um importante indicador de avaliação da saúde dos trabalhadores e das condições que o trabalho é realizado, como também da política de recursos humanos da instituição e do serviço de atenção à saúde do trabalhador. ${ }^{28}$

O absenteísmo pode ser definido como a rejeição do empregado a falta de humanização das condições de trabalho. Distinguem absenteísmo voluntário de involuntário e destacam que turnover, faltas por acidente de trabalho e atrasos na chegada ao serviço não devem ser considerados como absenteísmo. Para os autores o absenteísmo deve ser considerado apenas aquelas faltas sem o aviso anterior, não programada e sem justificativa médica. ${ }^{29}$

\section{Características do Presenteísmo}

O termo presenteísmo apresenta definições adotadas por alguns autores possuem, em linhas gerais, os mesmos significados, apenas ressaltando mais um ou outro aspecto de acordo com a área de cada pesquisador e da sua origem europeia ou norte americana. ${ }^{6}$

Para demonstrar variedade conceitual, registra-se

Quadro 1. Variedade conceitual de Presenteísmo

\begin{tabular}{|c|c|}
\hline Definições & Autores \\
\hline Comparecer ao trabalho, em oposição ao absenteísmo. & (Smith, 1970) \\
\hline Exibindo excelente assiduidade. & (Canfield \& Soash, 1955; Stolz 1933) \\
\hline $\begin{array}{c}\text { Horas de trabalho elevadas, aumentando a sua } \\
\text { visibilidade, mesmo que seja impróprio. }\end{array}$ & (Simpson, 1998; Worall et al, 2000) \\
\hline $\begin{array}{c}\text { Ser resistente em trabalhar em tempo parcial, preferindo } \\
\text { tempo integral. }\end{array}$ & (Sheridan 2004) \\
\hline $\begin{array}{c}\text { Não estar saudável, mas não apresentar absenteísmo por } \\
\text { doença. }\end{array}$ & (Kivimäki et al, 2005) \\
\hline $\begin{array}{c}\text { Ir para o trabalho apesar de se sentir doente. } \\
\text { (Aronsson, et a., 2000; Dew et al, 2005) }\end{array}$ \\
\hline $\begin{array}{c}\text { Ir para o trabalho apesar de se sentir doente ou estar } \\
\text { passando por outros acontecimentos que poderiam levar } \\
\text { a doença. }\end{array}$ & (Evans, 2004; Johansson \& Lundberg, 2004) \\
\hline $\begin{array}{c}\text { Redução da produtividade no trabalho devido a } \\
\text { problemas de saúde. }\end{array}$ & (Turpin et al, 2004) \\
\hline $\begin{array}{c}\text { Redução da produtividade no trabalho devido a } \\
\text { problemas de saúde ou outros eventos que atrapalham a } \\
\text { plena produtividade. }\end{array}$ & (Hummer, Sherman\& Quinn, 2002; Whitehouse, 2005) \\
\hline
\end{tabular}

Fonte: Jhons (2010) 
segundo quadro 1 onde enumerou-se nove definições diferentes de presenteísmo.

Em um estudo realizado pelo Institute for Health and Productivity Studies- IHPS, dos Estados Unidos, foram analisados os sintomas mais comuns do Presenteísmo: dores musculares, cansaço, ansiedade, angústia, irritação, depressão, insônia e distúrbios gástrico, sendo o estresse o grande gerador do Presenteísmo. Apontaram que os oito países mais estressados do mundo, em ordem decrescente, são: Japão (70\%), Brasil (30\%), China (24\%), Estados Unidos (20\%), Israel (18\%), Alemanha (16\%), França (14\%) e Hong Kong (12\%). No Brasil, o IHPS (2010) demonstra que três em cada dez brasileiros apresentam problemas de saúde devido ao estresse no trabalho. ${ }^{5}$

Durante muitos anos a expressão presenteísmo foi utilizada para ser apenas como o oposto do absenteísmo ou para ressaltar bom desempenho do trabalhador no seu emprego. ${ }^{6}$ A partir da década de 1980 os conceitos passaram a sofrer modificações seguindo a visão e o interesse dos pesquisadores de acordo com sua área. ${ }^{4,27}$

\section{Causas do Presenteísmo}

Trabalhar sob pressão está diretamente relacionado ao presenteísmo, quanto maior a pressão trabalhista, maior o nível do presenteísmo. ${ }^{29}$ Outros fatores relacionados ao alto presenteísmo são:

[...] sexo masculino, juventude, baixa autonomia ou extremamente alto e tem baixa segurança no emprego. Há de saber que o presenteísmo é baixo entre os trabalhadores que não foram contratados em tempo integral, com um baixo número de horas (oito horas ou menos por semana) e também entre os trabalhadores satisfeitos com seu salário. ${ }^{29: 31}$

As principais causas do presenteísmo são devidas a problemas do foro mental (depressão e estresse), músculos-esqueléticos (lombalgias e artrites) e respiratórios (constipações, síndromes gripais, asma) entre outras causas, $\mathrm{O}$ autor também relata causas definidas por outros autores: cefaleias/enxaquecas, alergias, refluxo gastresofágico, dermatites, hiperplasia benigna da próstata, diabetes mellitus, dismenorreia, dispepsia. Além destas causas o autor considera ainda os efeitos secundários de determinados medicamentos: os anti-histamínicos e as benzodiazepinas. ${ }^{30}$

As maiores taxas de presenteísmo encontramse nos enfermeiros, pois os mesmos referem que vão trabalhar com síndromes gripais, dores, problemas familiares relacionados com crianças ou idosos, problemas domésticos, depressão, preocupações e/ou ansiedade frequentemente. ${ }^{30}$

Todas estas condições afetam a produtividade, tanto em termos de qualidade e em quantidade do trabalho. O desempenho fica prejudicado em termos de qualidade porque podem ocorrer erros, e relativamente à quantidade, porque há uma repetição sistemática na execução das tarefas, devido à dificuldade de concentração. ${ }^{30}$

\section{Presenteísmo x Enfermagem}

A enfermagem é uma das profissões que tem elevados índices de presenteísmo e faz uma associação ao absenteísmo por doença e os baixos salários com o presenteísmo, desequilibrando as equipes de trabalho, trazendo consequências negativas para a instituição e para a assistência prestada. ${ }^{2}$

[...] ao analisar o presenteísmo na enfermagem, descreve que, nasúltimas décadas, os enfermeirosemambienteshospitalares se vêem obrigadosa fazer as coisascada vez melhor, com mais rapidez e com um número menor de pessoas, tendo que fazer "malabarismos" para conciliar o trabalho com as questões pessoais e familiares, o que deixa muito deles com muito pouco tempo para cuidar da sua própria saúde. ${ }^{2: 29}$

O grupo profissional da enfermagem encontra-se entre os grupos de risco para o presenteísmo, devido as suas extensas jornadas de trabalho e que o grau de compromisso que os profissionais que trabalham com enfermos adquirem levando-os a evitar ausências no trabalho. A mesma autora destaca a necessidade de unir esforços para melhorar as condições do trabalho hospitalar, diminuir o estresse no trabalho, melhorar a capacidade de prestação de uma assistência de qualidade e manter a segurança e a saúde dos profissionais da classe para a garantia de um cuidado de qualidade aos pacientes hospitalares, é fundamental manter a força de trabalho e a produtividade da enfermagem. ${ }^{2,28}$

\section{Discussão}

O profissional de acordo com a revisão de literatura inicialmente apresenta sinais e sintomas relacionados ao estresse, que se não tratados poderá acarretar a Síndrome de Burnout, que por sua vez fará com que o profissional adoeça, não consiga dar o seu melhor e com isso os problemas gerenciais e assistenciais começarão aparecer devido a insatisfação e a sobrecarga de trabalho no profissional e em toda a equipe caracterizando assim o presenteísmo, que se não tratado, evoluirá ao absenteísmo, caracterizado pela ausência do colaborador ao trabalho.

O presenteísmo ocorre quando o trabalhador mesmo doente comparece e/ou permanece no trabalho, realizando as atividades inerentes aos seus cargos, colocando em risco sua integridade física e também comprometendo a assistência prestada ao cliente, não apresentando um bom desempenho, mas problemas físicos e psicológicos.

A enfermagem, por sua vez, lida a todo tempo 
com perdas, sofrimento, emoções de terceiros, fazendo com que os profissionais sejam obrigados a the dar com toda essa mistura de sentimentos a todo o momento. O profissional por sua vez sobrecarrega-se, uma vez que além dos sentimentos deles tem que saber lhe dar, respeitar e muita das vezes ser o apoio que o cliente ou familiar precisa naquele momento.

Profissionais da enfermagem que trabalham em Unidades de Terapia Intensiva esbarram a todo tempo nesta problemática, uma vez que precisam trabalhar, mas nem sempre estão em condições psicológicas e físicas para encararem uma jornada de trabalho. Ficam com medo de faltar serviço e ser visto com maus olhos perante a chefia e aos próprios colegas de trabalho e justificam a sua falsa presença com "Se eu faltar, posso ser mandado embora", "Se eu faltar vou prejudicar e sobrecarregar a equipe". Mas até onde essa ida e permanência ao trabalho é benéfico para o(a) enfermeiro(a) e sua equipe?

$\mathrm{Na}$ maioria das vezes tem-se a falsa impressão de que uma equipe que não falta, é uma equipe boa. Esquece-se que os trabalhadores são humanos e também possuem sentimentos, e que estão vinte e quatro horas na beira do leito, participando de todo o sofrimento enfrentado pelo cliente e seus familiares durante $o$ tempo em que se encontra hospitalizado.

O presenteísmo sempre estará presente, devendo ser considerado um problema gerencial e assistencial, uma vez que traz consequências assistenciais e gerenciais tais como: baixo rendimento de produtividade, insatisfação do colaborador, vontade de abandonar o serviço, mudança de clima organizacional na equipe, maior índice de erros, tendência de desenvolver o absenteísmo, estresse profissional, má qualidade da assistência prestada, conflitos entre os componentes da equipe, insegurança, horas extras, levando ao adoecimento do profissional.

Os empregadores e os responsáveis organizacionais não conseguem ver o presenteísmo como problema na maioria das vezes, pois enxergam apenas a produção da equipe e a lucratividade da instituição, esperando sempre que as metas sejam alcançadas ao final do mês, mas esquecem da saúde dos seus profissionais, obrigando a trabalharem mesmo sem condições físicas e psicológicas para exercerem suas atividades e contribuir com a equipe para uma assistência de qualidade.

Uma má gestão de enfermagem acarreta em uma insatisfação dos profissionais envolvidos na assistência em com isso os profissionais vão para os seus postos de trabalhado insatisfeitos, e acabam machucandose, tratando mal os clientes que estão hospitalizados repercutindo em alguns casos no processo saúde-doença da pessoa, aumentando o tempo de hospitalização, uma vezquepalavrasmal ditas podem interferirnopsicológico do doente e atrapalhar no prognóstico do mesmo e por fim afastando-se de suas funções sobrecarregando toda a equipe, fazendo com que os demais profissionais da equipe venham-se ausentar posteriormente.

Todas as condições vão influenciar a saúde do trabalhador, sejam elas, organizacionais, doenças crônicas, doenças agudas, presenteísmo, absenteísmo, estresse, Burnout. Os custos do presenteísmo por não serem facilmente calculados, como no caso do absenteísmo, trazem uma maior preocupação, neste caso o profissional permanece em seu local de trabalho, mas não consegue produzir completamente a sua carga de trabalho.

\section{Conclusão}

De acordo com o estudo realizado a relevância do tema frente às organizações de saúde, mostrando os fatores que fazem os profissionais a adoecerem e, consequentemente, afastar-se do trabalho. Mostrase também a necessidade de maiores estudos sobre a temática no âmbito da enfermagem afim de avaliar o bem estar e a produtividade dos colaboradores.

Os enfermeiros assistenciais e gestores têm um importante papel na sensibilização de toda a equipe de enfermagem, pois são estes que assumem um papel de cuidar daqueles que prestam assistência direta aos pacientes, já que devem ter uma maior atenção para os hábitos de vida de todos os componentes de sua equipe, assim para sinais e sintomas de um possível adoecimento de seus componentes, procurando entender a causa e estar encaminhando-o para tratamento e resolução dos problemas identificados.

Cabe, então, a reflexão de que os agravos a saúde podem trazer limitações físicas e mentais pelos próprios sintomas, impossibilitando os colaboradores a comparecerem ao trabalho ou comparecerem mesmo sem condições exercerem suas atividades.

O foco no presenteísmo torna-se, então, uma estratégia que os enfermeiros gestores e assistencialistas devem adotar para promover o bem estar, a saúde, melhorar a produtividade, reduzindo assim os custos, ajudando assim na melhoria da assistência prestada.

O estudo é uma análise inicial onde se pretende investir em pesquisas futuras, na pós graduação, inclusive em pesquisas de campo, análise comparativa, tendo em vista a importância do tema na área de enfermagem.

\section{Referências}

1. Shimabuku RH. Presenteísmo à luz do modelo demandacontrole: um estudo moderacional. Pontifícia Universidade Católica de Goiás, Programa de Pós-Graduação Stricto Sensu em Psicologia, Goiânia, 2016.

2. Paschoalin HC, Griep RH et al. A produção científica sobre o presenteísmo na enfermagem e suas repercussões no cuidado. Rev APS. 2012 jul/set; 15(3):306-311. 
3. Middaugh D.J. Presenteeism: Sick and tired at work. Dermatology Nursing. April, 2007; 19(2):172-185.

4. Johns Gary. Presenteeism in the workplace: A review and research agenda. Journal of Organizational Behavior. 2010; 31(4): 519-542.

5. Silva AM, Ferreira RP, Sassi RJ. A qualidade de vida no trabalho versus absenteísmo e presenteísmo. Rio de Janeiro- Brasil, 12 e 13 de agosto de 2010. Disponível em: https://www.marinha.mil.br/spolm/sites/www. marinha.mil.br.spolm/files/73775.pdf. Acessado em 25/03/2018.

6. Ferraz F, Hecksher S, Carvalho E. Presenteísmo: as perdas diárias e silenciosas. Área temática: Gestão De Segurança no Trabalho e Ergonomia. INOVARSE - XII Congresso Nacional de Excelência em Gestão \& III INOVARSE - Responsabilidade Social Aplicada. Set. 2016.

7. Johansen V, Aronsson, G, Marklund S. Positive and negative reasons for sickness presenteeism in Norway and Sweden: a cross-sectional survey. BMJ open, 2014;4(2).

8. Godengerg M. A arte de pesquisar. Rio de Janeiro: Record, 1997. Disponível em: http://www.ufrgs.br/cursopgdr/downloadsSerie/derad005. pdf. Acessado em 25/03/2018.

9. Córdova F P. Método de pesquisa. Porto Alegre: Editora da UFRGS, 2009. Disponível em: http://www.ufrgs.br/cursopgdr/ downloadsSerie/derad005.pdf. Acessado em 25/03/2018.

10. Lehfeld, N A Souza, Barros, A J P. Fundamentos de Metodologia Científica. $3^{\mathrm{a}}$ edição, editora Makron, 2007, Brasil. Disponível em: http:// posgraduando.com/diferencas-pesquisa-descritivaexploratoria-explicativa/. Acessado em: 25/03/2018.

11. Perovano DG. Manual de Metodologia Científica para a Segurança Pública e Defesa Social. 1a edição, editora Jurua, 2014, Brasil. Disponível em: http://posgraduando.com/diferencas-pesquisa-descritiva-exploratoriaexplicativa/. Acessado em: 25/03/2018.

12. Brasil. Ministério da Saúde. Conceitos de Doença. Dispinível em: https://www.portaleducacao.com.br/conteudo/artigos/esporte/conceitos-dedoenca/44118. Acessado em 23/12/2017.

13. Brasil. Ministério da Saúde. Política Nacional de Saúde do Trabalhador. Disponível em: http://www.saude.mt.gov.br/cosat/pagina/256/ politica-nacional-s-t. Acessado em 27/12/2017.

14. Brasil. Ministério da Saúde. Política Nacional visa à redução de acidentes e doenças do trabalho. Disponível em: http://www.brasil.gov.br/ saude/2012/08/politica-nacional-visa-a-reducao-de-acidente-e-doencas-dotrabalho. Acessado em 27/12/2017.

15. Vianna, L A P. Processo Saúde-Doença. Módulo Político Gestor. Disponível em: http://www.unasus.unifesp.br/biblioteca_virtual/esf/1/ modulo politico gestor/Unidade 6.pdf. Acessado em 27/12/2017

16. Gomes H, Jesus AG. Síndrome de Burnout em Enfermeiros. 2012;4(6). Jornada Científica da UNIVERSO - Suplemento Saúde Brasil

17. Gonçalves RMV, Schneider KS. Estratégias de Enfrentamento da Síndrome de Burnout na Enfermagem. Caderno de Saúde e Desenvolvimento, jan 2016;8(5)

18. Dalmolin et AL. Implicação do sofrimento moral para os(as) enfermeiros(as) e aproximações com o Burnout. Revista texto e Contexto Enfermagem, Florianópolis, jan-mar 2012; 21(1):200-208. Disponível em: $<$ http:/www.lilacs.br $>$. Acessado em 27/12/2017.

19. Marinho, Rita de Cássio. Estresse ocupacional, estratégia de enfrentamento e Síndrome de Burnout: um estudo em um hospital. Dissertação (mestrado) da Universidade de Taubaté. Departamento de Economia, Contabilidade e Administração. Taubaté-SP. 2005. 118f. Disponível em: http://scholar.google.com.br. Acessado em 27/12/2017.

20. Benevites - Pereira, AMT. Burnout: quando o trabalho ameaça a saúde do trabalhador. São Paulo: Casa do Psicólogo, 2002.

21. Seligmann-Silva. Psicopatologia e Saúde Mental no Trabalho. In: Mendes, R. Patologia do trabalho. $1^{\text {a }}$ ed. Rio de Janeiro, Atheneu. 2003.

22. Andrade LPV. O adoecimento dos profissionais de enfermagem no ambiente de trabalho: burnout uma revisão de literatura / Lygia Priscila Vieira de Andrade. Assis, Trabalho de conclusão do curso Enfermagem.
Educacional do Município de Assis-FEMA. 2015. 43p.

23. Oliveira EB, Souza NVM. Esforço e recompensa no trabalho do enfermeiro residente em unidades especializadas. Rev enferm UERJ. 2012; 20:457-62.

24. Faria AC, Barboza DB, Domingos NAM. Absenteísmo po transtornos mentais na enfermagem no período de 1995 a 2004. Arq Ciênc Saúde [periódico na internet] 2005 [citado em 13 jan 2013] 12:14-20. Disponível em: http://www.cienciasdasaude.famerp.br/Vol-12-1/03\%20 -\%20id\%20100.pdf. Acessado em 23/12/2017.

25. Bizarra MA, Balbino CM \& Silvino ZR. Segurança do paciente - o papel do enfermeiro no gerenciamento de risco focado na UTI. Revista Pró-UniverSUS. 2018 Jan./Jun.; 09 (1): 101-104. Disponível em: http:// editora.universidadedevassouras.edu.br/index.php/RPU/article/view/1232. Acessado em: 15/8/2018.

26. Penatti I, Zago JS, Quelhas O. Absenteísmo: As consequências na gestão de pessoas. III SEGeT - Simpósio de Excelência em Gestão e Tecnologia. Disponível em: http://inf.aedb.br/seget/artigos06/898_Seget Izidro\%20Penatti.pdf. Acessado em: 25/03/2018.

27. Bernstorff VH. Relações entre satisfação, competência, saúde e absenteísmo no trabalho de uma grande instituição bancária pública. Tese de doutorado - UNB, Dez - 2007.

28. Umann J, Guido LA, et al. Absenteísmo na equipe de enfermagem no contexto hospitalar. CiencCuidSaude 2011 Jan/Mar; 10(1):184-190

29. Chadwick-Jones JK, Nilcholson N e Brown C. Social psychology of absenteeism. New York: Praeger Publishers, 1982.

30. Flores-Sandí G. Presentismo: Potencialidad el accidentes de salud. AMC, 48(1), enero-marzo, 2006, pp. 30-34. Colegio de Médicos y Cirujanos de Costa Rica San José, Costa Rica. 